\title{
Analysis of Part Motion on a Longitudinally Vibrating Plate
}

\author{
Dan Reznik* \\ John Canny \\ Ken Goldberg \\ dreznikecs.berkeley.edu jfcecs.berkeley.edu goldberg@ieor.berkeley.edu \\ University of California-Berkeley \\ Berkeley, CA 94720 USA
}

\begin{abstract}
-
We analyze the dynamics of part motion for a novel type of planar parts feeder consisting of a longitudinally vibrating flat plate and a part placed on its surface. For each vibration cycle, the plate's velocity is held positive (forward motion) for a longer time than it is held negative (backward motion). This type of asymmetric vibration combined with the non-linear nature of Coulomb friction causes the part to accelerate along a straight line to a terminal velocity called the "feed rate". The average force exerted by the plate on the part is shown to be proportional to the latter's deviation from the feed rate. In other words, the part behaves as if it were immersed in a forward moving viscous fluid. Expressions for the feed rate and viscosity constants are derived with respect to various physical and control parameters. Rigid-body dynamic simulation results are shown to be in good agreement with the analysis.
\end{abstract}

\section{INTRODUCTION}

Frictional interactions of parts with a vibratory structure is the technique used by bowl feeders [1] to propel industrial parts along a helical track containing cleverly designed part-orienting features [2]. Each rigid vibration of the entire bowl is a combination of both translation and rotation, causing parts to hop, slide, stick, and inch forward along the track. Boothroyd [1] used this sequence of part motion "states" to derive an expression for the average speed - called the feed rate - at which parts move along the track.

While full $3 \mathrm{~d}$ vibration of a flat surface certainly accomplishes the goal of part transport, in this paper we show that the same can be accomplished through much simpler onedimensional, longitudinal vibrations of a flat plate. Namely, we consider the bare-bones "parts feeder" design shown in Figure 1 , consisting of a flat plate actuated by a linear motor. A small part is placed on the plate's surface. We assume that Coulomb friction [3] is the only force acting on the part and that the plate's motion is such that the part is always sliding over it (no sticking). Notice that the plate's 1d motion rules out part hopping. We avoid the problem of contact point indeterminacy [4] between the plate and the part's footprint by treating the latter as a point mass.

\footnotetext{
* Support provided in part by NSF Grant FD93-19412.
}

We exploit one interesting feature of the Coulomb frictional force: its magnitude is independent of the part's velocity relative to the plate. namely, we control the plate's oscillatory motion so that it spends more time moving forward (positive velocity) than backward (negative velocity). Imagine a part initially at rest on the plate's surface. During one such time-asymmetric vibration, the part will experience forward force of magnitude $\mu m g$ for a longer time than it will experience the same force on the opposite direction, resulting in net a forward momentum added to the part after the cycle. We show that rather than accelerating indefinitely, the part reaches a terminal velocity (the feed rate) whose value depends on both physical and control parameters. We also show that the average force exerted by the plate on the part is proportional to the latter's deviation from the feed rate, i.e., the part behaves as if it were immersed in a viscous fluid flowing forward at the feed rate.

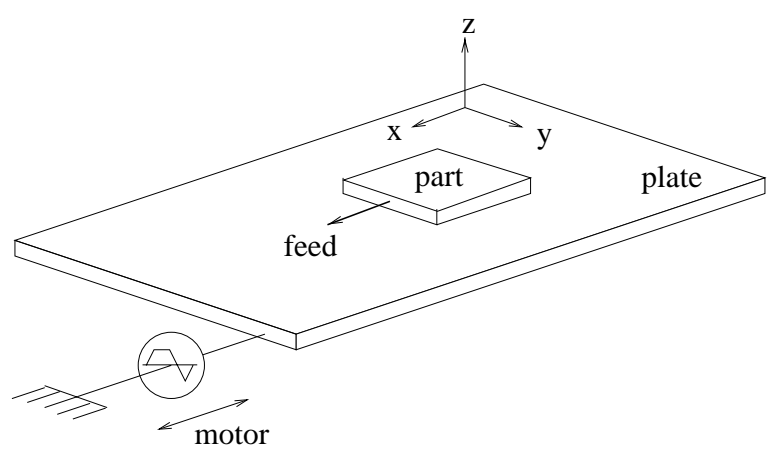

Fig. 1. Model of the longitudinally vibrating parts feeder. A linear motor commands in-plane, time-asymmetric oscillations of the plate, which cause a part resting on its surface to move forward at a constant velocity called the feed rate.

\section{A. Related Work}

The idea to use vibrations of a single plate to move parts on the plane has been investigated by Böhringer et al. [5]. In their case, the transversal vibrations of a metallic plate are used to gather small particles at the vibration nodes. Examples of part manipulation devices which exploit some form of timeasymmetric driving force include air flow nozzle arrays [6] and 
multi-phase electric field tubes [7]. Arrays of parallel actuators such as orientable rollers [8], thermoactuated cilia [9], and a torsional resonators $[10,11]$ have been proposed as novel designs for non-prehensile manipulation in the plane.

The remainder of this paper is organized as follows: In Section II we present the details of plate motion and control. In Section III we present a detailed analysis of the dynamics of part motion. In Section IV we show rigid-body dynamic simulations results which were used to validate the analysis. Section $\mathrm{V}$ presents conclusions and directions for further work.

\section{Plate Motion}

We assume the linear motor converts input voltage to output acceleration of the plate. The simplest input waveform which integrates to a velocity profile whose positive portion is longer than the negative one is the three-level wave shown in Figure 2(a). It causes the plate to accelerate in sequence at $\left\{a_{\text {max }}, 0,-a_{\text {max }}\right\} \mathrm{m} / \mathrm{s}^{2}$, assuming $a_{\text {max }}$ is feasible by the motor. The acceleration waveform is periodic with period $T$. Shown in Figure 2(b) is the plate's velocity profile, consisting of a positive (forward displacement) semicycle of length $F$ followed by a negative (backward displacement) semicycle of period $B$. The integral of this curve is the plate's displacement, shown in Figure 2(c).

\section{A. The no-net-motion constraint}

The positive semicycle is an isosceles trapezoid with parallel bottom and top sides of lengths $F$, and $P$, respectively. The trapezoid's height is the plate's maximum forward velocity, $\nu_{\max }$. The negative semicycle is an isosceles triangle of base $B$ and height $\left|\nu_{\min }\right|$, the plate's maximum backward velocity. The areas subtended by the positive and negative semicycles are labeled $S_{f w d}$ and $S_{b w d}$, respectively. Stating that the net plate displacement per cycle should be zero constrains $S_{f w d}=S_{b w d}$. From simple geometry:

$$
\begin{aligned}
S_{f w d} & =\frac{F+P}{2} \frac{\nu_{\max }}{2} \\
S_{b w d} & =\frac{B\left|\nu_{\min }\right|}{2}
\end{aligned}
$$

But $\nu_{\max }=a_{\max } t_{1}$, where $t_{1}=(F-P) / 2$ as indicated in Figure 2(a). From Figure 2(b), $\nu_{\min }=-a_{\max } B / 2$. So the equal area constraint implies:

$$
a_{\max } \frac{(F+P)}{2} \frac{(F-P)}{2}=a_{\max } \frac{B}{2} \frac{B}{2}
$$

And consequently $B^{2}=F^{2}-P^{2}$, which yields:

$$
P=\sqrt{F^{2}-B^{2}}
$$

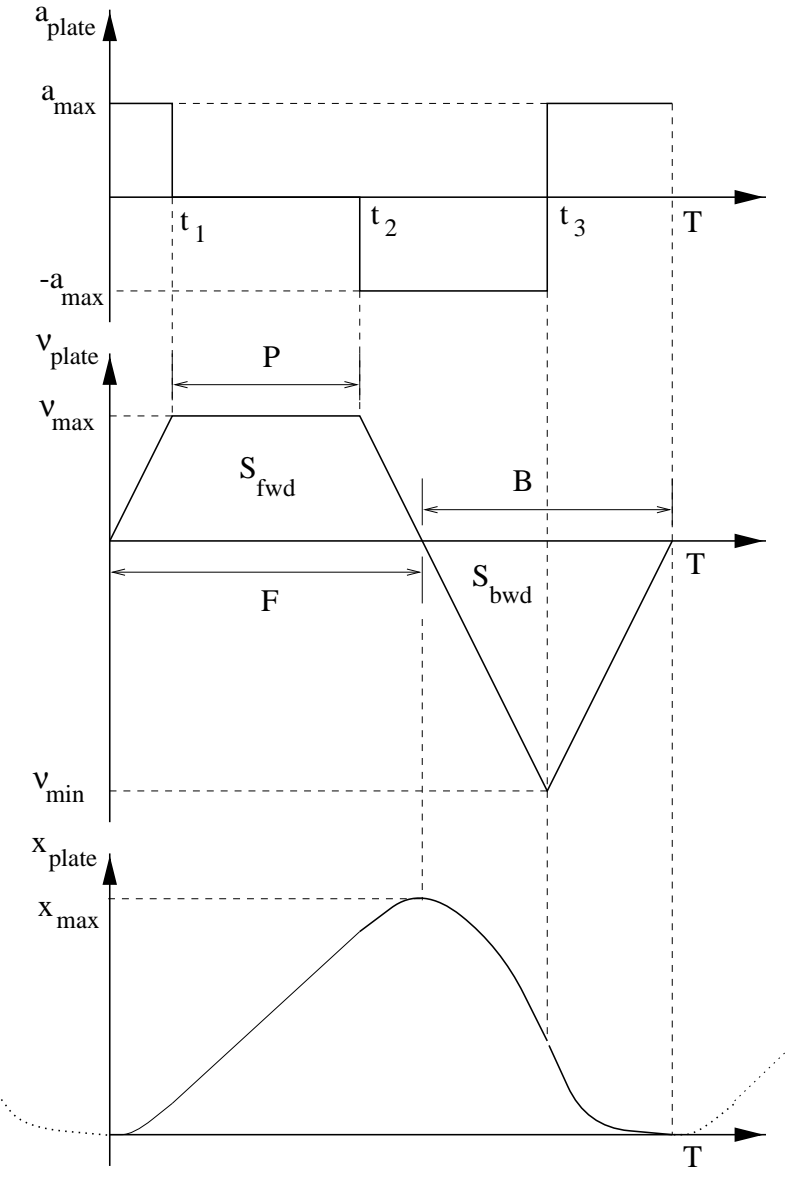

(a)

(b)

Fig. 2. Details of plate motion. (a), (b), and (c) show respectively one cycle of the plate's acceleration, velocity, and position.

Noting that the period $T=F+B$, we call $d=F / T$ the duty cycle of the velocity profile. Relations $F=T d$ and $B=$ $T(1-d)$ substituted on Equation 1 yield an expression for $P$ as a function of $T$ and $d$ :

$$
P=T \sqrt{2 d-1}
$$

Note: from now on all quantities will be derived in terms of the independent design parameters $T, d$, and $a_{\max }$.

\section{B. Plate's maximum displacement}

To derive the peak-to-peak plate displacement per cycle, shown as $x_{\text {max }}$ in Figure 2(c), we integrate the forward semicycle of the velocity profile:

$$
\begin{aligned}
x_{\text {max }} & =\int_{0}^{F} \nu_{\text {plate }}(t) d t \\
& =\int_{0}^{t_{1}} a_{\text {max }} t+\int_{t_{1}}^{t_{2}} \nu_{\text {max }}+\int_{t_{2}}^{F} a_{\text {max }}(T-t)
\end{aligned}
$$


Noting that:

$$
\begin{aligned}
t_{1} & =\frac{F-P}{2}=\frac{T}{2}(d-\sqrt{2 d-1}) \\
t_{2} & =\frac{F+P}{2}=\frac{T}{2}(d+\sqrt{2 d-1}) \\
\nu_{\text {max }} & =a_{\text {max }} t_{1}=a_{\text {max }} \frac{T}{2}(d-\sqrt{2 d-1})
\end{aligned}
$$

So Equation 3 evaluates to:

$$
x_{\text {max }}=a_{\text {max }}\left[\frac{T(1-d)}{2}\right]^{2}
$$

\section{PART DYNAMICS}

Here we study the motion of a small part of mass $m$ idealized as a point mass. The plate's vibratory motion gives rise to an instantaneous frictional force $f_{\text {fric }}$ acting on the part. The Coulomb friction model (used here) states that $f_{\text {fric }}$ is (i) in the direction of the plate's motion relative to the part, and (ii) of magnitude $\mu \mathrm{mg}$, where $g=981 \mathrm{~cm} / \mathrm{s}^{2}$ is the acceleration of gravity and $\mu$ is the coefficient of friction of the plate/part interface. We assume the part is always sliding over the plate.

\section{A. Quasi-static analysis}

The following quasi-static assumption (which will be dropped later) aids in understanding the feeder's principle of operation: the part's change in velocity within one vibration cycle is assumed negligible. In the worst case, the part will accelerate monotonically at a rate of $\mu g$ during the entire period $T$. In terms of the plate's maximum velocity, this assumption can be stated as:

$$
\mu g T<<\nu_{\max }
$$

A given part velocity $\nu_{\text {part }}$ splits the velocity profile $\nu_{\text {plate }}(t)$ into two portions: (i) $\nu_{\text {plate }}>\nu_{\text {part }}$, (ii) $\nu_{\text {plate }}<\nu_{\text {part }}$. This can be visualized by superimposing $\nu_{\text {part }}$ (a horizontal line according to quasi-statics) over the $\nu_{\text {plate }}$ graph, as illustrated in Figure 3. Denote phase (i)'s duration by $t_{f w d}$; phase (ii)'s duration is then simply $T-t_{f w d}$. During (i), $f_{\text {fric }}=\mu m g$, and during (ii) $f_{\text {fric }}=-\mu m g$. The main idea is that the part will experience a net positive (resp. negative) change in momentum over the entire cycle if $t_{f w d}>T / 2\left(\right.$ resp. $\left.t_{f w d}<T / 2\right)$, i.e., the $\nu_{\text {part }}$ horizontal line slides up (resp. down) over the velocity profile, Figure 3, resulting in a narrower (resp. wider) cross-section of the positive trapezoid. This suggests that the part will stop accelerating at an equilibrium velocity $\nu_{e q}$ such that $t_{f w d}=T / 2$.

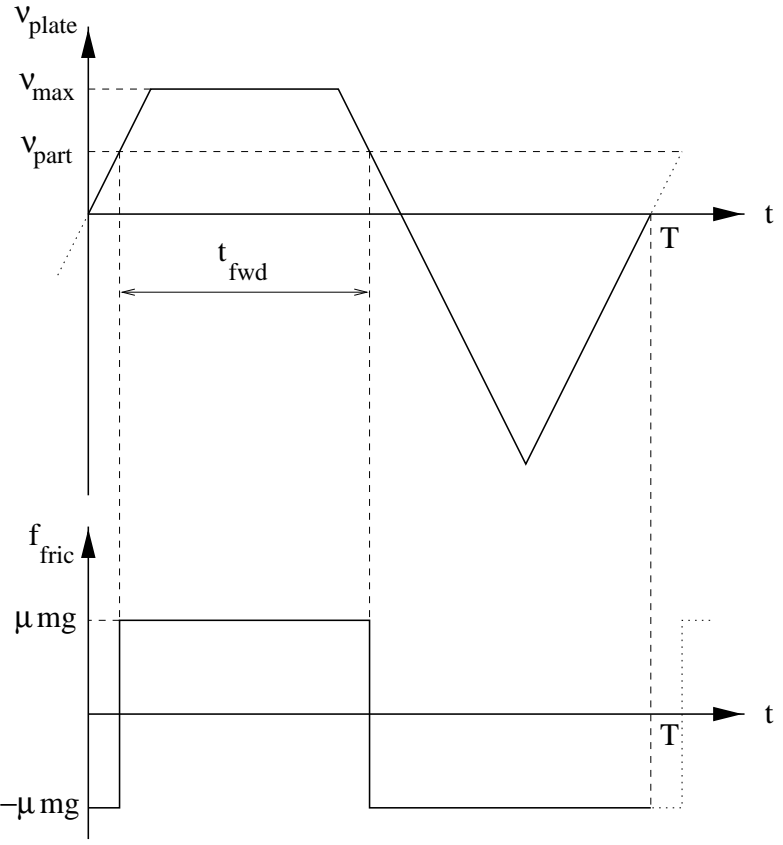

Fig. 3. Frictional forces acting on the part. The top diagram shows the plate's velocity profile superimposed on the part's velocity (assumed constant during the cycle). The bottom graph shows that while $\nu_{\text {plate }}>\nu_{\text {part }}$ (which lasts for $t_{f w d}$ ), the frictional force $f_{\text {fric }}=$ $\mu m g$; during the rest of the cycle $\nu_{\text {plate }}<\nu_{\text {part }}$ and therefore $f_{\text {fric }}=-\mu m g$. In the cycle shown, the part will de-accelerate since $t_{\text {fwd }}<T / 2$.

1) Saturation: If $P>T / 2$, the part will accelerate to $\nu_{\text {max }}$ and reach a saturation point after which it can no longer accelerate $^{1}$. From Equation 2, we derive the maximum duty cycle $d_{s a t}$ for which $\nu_{e q}<\nu_{\max }$ :

$$
d_{\text {sat }}=\frac{5}{8}=0.625
$$

For $d<d_{s a t}, \nu_{e q}$ is derived as follows: let $r$ denote the ratio $\nu_{e q} / \nu_{\text {max }}$, which ranges from 0 to 1 . Then, from the trapezoidal shape of the positive velocity semicycle, $t_{f w d}$ is the linear combination:

$$
t_{f w d}=F(1-r)+P r
$$

Equating the above to $T / 2$, and solving for $r$ and $d$ yields:

$$
\begin{array}{r}
r=\frac{2 d-1}{2(1-d)^{2}}(d+\sqrt{2 d-1}) \\
d=\frac{r(1+2 \sqrt{r})-1 / 2}{r(3+2 \sqrt{r})-1}
\end{array}
$$

An "optimal" value for $r$ is $1 / 2$, since it is places $\nu_{e q}$ midrange between 0 (no feeding velocity) and $\nu_{\max }$ (the saturation point). Plugging $r=1 / 2$ on Equation 8 yields:

\footnotetext{
${ }^{1}$ Notice that at the saturation speed $\nu_{e q}=\nu_{\text {max }}$, the average frictional force over a cycle will change abruptly if the part's velocity is slightly increased.
} 


$$
d_{\text {opt }}=2-\sqrt{2} \cong 0.586
$$

Also, using $r=1$ (the saturation ratio) in Equation 8 yields $d=0.625$, which coincides with the value computed for $d_{s a t}$, above.

To derive $\nu_{e q}$ in terms of $d$, use $\nu_{e q}=r \nu_{\text {max }}$, Equations 7 and 4, yielding the following linear relationship:

$$
\nu_{e q}=\frac{a_{\max } T}{4}(2 d-1)
$$

Notice that for $d=0.5, \nu_{e q}=0$, i.e., as expected the part doesn't feed since the velocity profile is perfectly symmetric, with $P=0$, and $t_{f w d}=T / 2$.

As stated above, if $d>d_{s a t}, \nu_{e q}$ saturates (i.e., tracks) $\nu_{\text {max }}$. We are now ready to formulate an expression for the equilibrium velocity over the entire $d$ range ( 0.5 to 1.0 ):

$$
\nu_{e q}= \begin{cases}\frac{a_{\max } T}{4}(2 d-1) & 0<d<5 / 8 \\ \frac{a_{\max } T}{2}(d-\sqrt{2 d-1}) & 5 / 8<d<1\end{cases}
$$

Notice that for $d=5 / 8, \nu_{e q}$ is maximal and equals $a_{\max } T / 16$. The graph for this expression in terms of the duty cycle is shown in Figure 4(a), for $a_{\max }=T=1$.

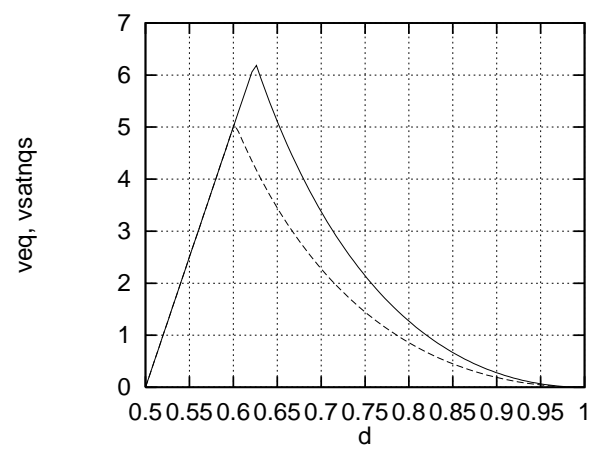

Fig. 4. The part's feed rate $\nu_{e q}$ plotted against the duty cycle $d$, assuming $a_{\max }=T=1$. (a) Quasi-static assumption (solid line): $\nu_{e q}$ is linear up to $d=0.625$ after which it tracks $\nu_{\max }$. (b) Nonquasi-static feed (dotted line): Non-quasi-static saturation occurs before the quasi-static one; from that point the non-quasi-static feed rate is strictly less than the quasi-static one.

2) A model for quasi-static friction: At the quasi-static equilibrium feeding velocity, the total force applied to the part per cycle is zero. If an external force $f_{e x t}$ is applied to the part in the $\mathrm{X}$ direction, what should its resulting velocity be? The part will seek a new equilibrium velocity $\nu^{\prime}$ such that $f_{\text {fric }}=f_{e x t}$. Denote the portion of the velocity profile for which $\nu_{\text {plate }}>\nu^{\prime}$ by $t_{f w d}^{\prime}$. Referring back to Figure 3, the equilibrium equation becomes:

$$
\frac{\mu m g}{T}\left[t_{f w d}^{\prime}-\left(T-t_{f w d}^{\prime}\right)\right]=f_{e x t}
$$

Let $r^{\prime}$ denote the new ratio $\nu^{\prime} / \nu_{\text {max }}$. Then $t_{f w d}^{\prime}$ is the following linear combination of $F$ and $P$ :

$$
t_{f w d}^{\prime}=F\left(1-r^{\prime}\right)+P r^{\prime}
$$

Which allows us to derive the following relationship:

$$
\begin{aligned}
f_{e x t} & =-K\left(\nu^{\prime}-\nu_{e q}\right) \text { with: } \\
K & =\frac{4 \mu m g}{a_{\max } T}
\end{aligned}
$$

The above equation states that around a neighborhood of $\nu_{e q}$ the plate's frictional force (averaged over sufficiently many cycles) is proportional to the part's velocity deviation from the feed rate, i.e., the part behaves as if it were immersed in a viscous fluid moving forward at the feed rate (where $K$ is the constant of viscosity).

\section{B. Non-quasi-static analysis}

When the quasi-static assumption of Equation 6 does not hold, the velocity of the part within each vibration cycle can be expected to vary appreciably with respect to the plate's, as shown in Figure 5. As stated above, the part will accelerate (or deaccelerate) at a constant rate equal to $f_{\text {fric }} / m$, i.e., $\mu g$.

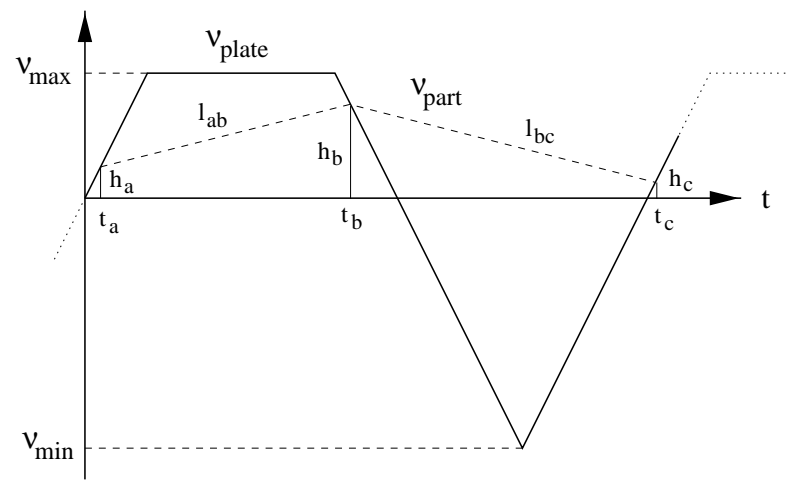

Fig. 5. Part acceleration within each cycle. Assume part accelerates at a rate of $\mu g$ (resp. $-\mu g$ ) when its velocity is less (resp. more) than the plate's.

Without loss of generality, we will track the part's velocity starting from a value $h_{a}$ along the left edge of the positive semicycle. This edge is described by the equation $\nu_{a}(t)=a_{\text {max }} t$, and we set $\nu_{a}\left(t_{a}\right)=h_{a}$, so $t_{a}=h_{a} / a_{\max }$. The part's constant acceleration within the positive semicycle is represented by a line $l_{a b}(t)=\mu g t+c_{1}$; with the constraint $l_{a b}\left(t_{a}\right)=h_{a}$ derive:

$$
c_{1}=\frac{h_{a}}{a_{\max }}\left(a_{\max }-\mu g\right)
$$

This line meets the right edge of the positive semicycle at $\left(t_{b}, h_{b}\right)$. That edge's equation is $\nu_{b}(t)=a_{\max }(T d-t)$, and $h_{b}=\nu_{b}\left(t_{b}\right)$. The intersection of this edge with $l_{a b}$ occurs at: 


$$
\begin{aligned}
t_{b} & =\frac{a_{\max } T d-c_{1}}{a_{\max }+\mu g} \\
h_{b} & =a_{\max }\left(T d-t_{b}\right)
\end{aligned}
$$

Beyond the intersection, $\nu_{\text {plate }}$ becomes smaller than $\nu_{\text {part }}$ causing the part to de-accelerate. This is represented by a line $l_{b c}(t)=-\mu g+c_{2}$. Setting $l_{b c}\left(t_{b}\right)=h_{b}$ yields:

$$
c_{2}=h_{b}+\mu g t_{2}
$$

Line $l_{b c}$ will intersect the left edge of the next positive semicycle at $\left(t_{c}, h_{c}\right)$. This edge is described by the equation $\nu_{c}(t)=$ $a_{\text {max }}(t-T)$, so $h_{c}=\nu_{c}\left(t_{c}\right)$. By imposing $l_{b c}\left(t_{c}\right)=h_{c}$ we compute the aforementioned intersection. Then:

$$
\begin{aligned}
t_{c} & =\frac{a_{\max } T+c_{2}}{a_{\max }+\mu g} \\
h_{c} & =a_{\max }\left(t_{c}-T\right)
\end{aligned}
$$

By backward substitution, $h_{c}$ can be expressed as a linear function of $h_{a}$ :

$$
\begin{aligned}
h_{c} & =k_{1} h_{a}+k_{2} \text { with: } \\
k_{1} & =\left(\frac{a_{\max }-\mu g}{a_{\max }+\mu g}\right)^{2} \\
k_{2} & =\frac{a_{\max } \mu g T}{\left(a_{\max }+\mu g\right)^{2}}\left[a_{\max }(2 d-1)-\mu g\right]
\end{aligned}
$$

At the end of every cycle $h_{c}$ "becomes" a new $h_{a}$, so Equation 12 is really a recurrence relation with $h_{c}=h_{a}$. Since the recurrence's gain $k_{1}$ is positive and strictly less than unity, the recurrence converges to the solution of $h_{a}=k_{1} h_{a}+k_{2}$, yielding:

$$
h_{a, \text { stable }}=\frac{k_{2}}{1-k_{1}}=\frac{T}{4}\left[a_{\max }(2 d-1)-\mu g\right]
$$

By using the above expression for $h_{a}$ to compute $c_{1}$ in Equation 11 obtain:

$$
h_{b, \text { stable }}=\frac{T}{4}\left[a_{\max }(2 d-1)+\mu g\right]
$$

The average velocity of the part within a cycle is the average within the acceleration portion plus the average within the deacceleration portion, but both these straight lines span the $h_{a}$ to $h_{b}$ interval, so the average velocity $\nu_{e q}$ for the entire cycle is simply $\left(h_{a, \text { stable }}+h_{b, \text { stable }}\right) / 2$. With Equations 13 and 14 get:

$$
\nu_{e q, n q s}=\frac{a_{\max } T}{4}(2 d-1)
$$

Where "nqs" stands for non-quasi-static. This result matches the expression for $\nu_{e q}$ derived under the quasi-static assumption (Equation 9), so surprisingly, at equilibrium, part acceleration and de-acceleration phenomena cancel each other out in the sense of per-cycle average velocity.

1) Dynamic (non-quasi-static) saturation: In the quasistatic model, saturation occurs when the plateau duration $P$ is larger than half the period $T$. The part accelerates until it hits the $\nu_{\max }$ hard limit. In the non-quasi-static case, the onset of saturation occurs when the part's acceleration within the positive semicycle takes $\nu_{\text {part }}$ to the upper right corner of that semicycle, as shown in Figure 6. To use the notation of Figure 5, this corresponds to $h_{b}=\nu_{\max }$, which with Equations 4 and 14 can be solved for the $d$ above which the system saturates:

$$
d_{s a t, n q s}=\frac{5 a_{\max }^{2}+\mu g\left(\mu g-2 a_{\max }\right)}{8 a_{\max }^{2}}
$$

Notice that if $\mu g$ is small compared to $a_{\max }$ (another phrasing of the quasi-static assumption, Equation 6), then $d_{s a t, n q s}$ reduces to $5 / 8$, which matches the saturation duty cycle for the quasi-static case. The value of $d_{s a t, n q s}$ is plotted against $a_{\max }$ in Figure 7.

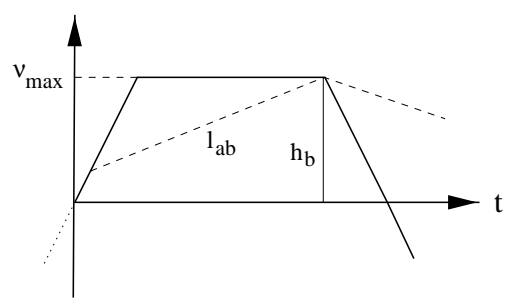

Fig. 6. The onset of saturation in the non-quasi-static case. This occurs when part acceleration in the positive semicycle takes part velocity to the upper right corner of that semicycle.

Equation 16 can be solved for the ratio $a_{\max } / \mu g$ to give the following alternative characterization of the onset of saturation:

$$
\frac{a_{\max }}{\mu g}>\frac{1+2 \sqrt{2 d-1}}{5-8 d}
$$

2) Beyond the saturation point: If the inequality in Equation 17 is strictly satisfied, then the situation becomes as depicted in Figure 8, namely, part acceleration within the positive semicycle hits the $\nu_{\max }$ hard limit before the end of the plateau phase, and the part follows the plate kinematically for a period of $L$ seconds, as shown in Figure 8 . This mean velocity can be computed as the following weighted average:

$$
\nu_{s a t}=\frac{1}{T}\left[L \nu_{\max }+(T-L) \frac{\left(\nu_{\max }+h_{a}\right)}{2}\right]
$$




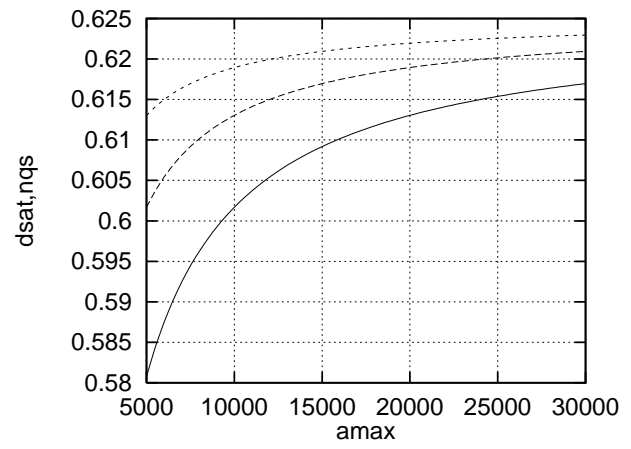

Fig. 7. Non-quasi-static saturation duty cycle $d_{\text {sat, nqs }}$ plotted against plate/motor maximum acceleration $a_{\max }$. The three curves correspond (from lowest in the graph to highest) to $\mu$ equal to $1.0,0.5$, and 0.25 , respectively. Notice that as $\mu$ decreases and/or $a_{\max }$ increases, the quasi-static $d_{\text {sat }}=0.625$ is approached.

Which makes use of the equilibrium relation $h_{a}=h_{c}$. It can be shown that $h_{a}$ will converge to a value yielding the following saturation feed rate:

$$
\begin{array}{r}
\nu_{\text {sat }}=\frac{a_{\max } T}{2}\left[\frac{a_{\max }-\mu g}{a_{\max }+\mu g}\right]^{2}(d-\sqrt{2 d-1}) \\
d>d_{\text {sat,nqs }}
\end{array}
$$

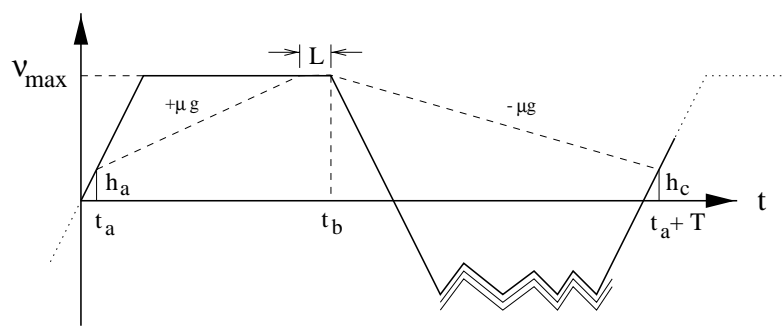

Fig. 8. The part's velocity profile beyond the non-quasi-static saturation point: the part accelerates rapidly enough to hit the $\nu_{\max }$ barrier before the end of the plateau phase.

A plot of the equilibrium velocity predicted by both models (quasi- and non-quasi-static) is presented in Figure 4(b). This Figure shows that beyond the saturation point (where the curves intersect) $\nu_{s a t}$ is strictly less than $\nu_{e q}$, meaning that non-quasistatic behavior reduces the feed rate beyond the saturation point $d_{s a t, n q s}$. Furthermore, the saturation point itself is strictly less than the quasi-static one, so the peak feed rate predicted by the quasi-static model is never reached.

\section{Simulation}

As a tool for validating the analytical results derived in the previous sections, we ran various rigid-body dynamic simulations using the Impulse tool [12]. A rectangular part $1 \times 1 \times 0.1 \mathrm{~cm}$ and mass $m=0.1 g$ was used though the analytical results were derived assuming the part was a point mass. The vibrating plate was defined as a one link robot with a single degree of freedom along the $\mathrm{X}$ axis. The acceleration wave described in Section II was implemented by associating an Impulse control law to the plate. The simulation studies conducted involved measuring the equilibrium feed rates with respect to (i) the duty cycle, and (ii) externally applied forces.

\section{A. Choosing design parameters}

A frequency of $50 \mathrm{~Hz}(T=.02 \mathrm{~s})$ was chosen for the plate, corresponding to the typical operating frequency of existing vibratory bowl feeders [1]. The plate's oscillation amplitude $x_{\max }$ was set at $1 / 4 \mathrm{~cm}$. At $d=0.586$, (for which $\nu_{e q}=\nu_{\max } / 2$ ), Equation 5 prescribes $a_{\text {max }} \cong 15,000 \mathrm{~cm} / \mathrm{s}^{2}$, i.e., approx. $15 \mathrm{~g}$. At this level, and using $\mu=1.0$, Equation 16 yields $d_{\text {sat }, n q s} \cong$ 0.61 , so at $d=0.586$ we are well in the non-saturating region.

\section{B. $\quad \nu_{e q} v s . d$}

Figure 9 shows a plot of the measured equilibrium velocity with the above parameters for $a_{\text {max }}$ set to 10,15 , and $2010^{3} \mathrm{~cm} / \mathrm{s}^{2}$. The data points are superimposed with the analytical curves derived in previous sections, showing good predictive behavior.

The pre-saturation values tend to be lower than the analytical results, and this was due to an anomaly in the numerical integration of Impulse, which is still being investigated. In particular, the simulator will occasionally apply impulses to the part with a negative x component, much higher than $a_{\text {max }}$, showing that the package has some numerical instability problems. When these anomalies are averaged out with the otherwise stable computations, they result in lower feed rates. The post-saturation values follow closely the non-quasi-static results, tailing off to very small feed rates as $d$ approaches unity.

\section{C. $\nu_{e q}$ vs. f fext}

The design parameters chosen above resulted in unstable computations for the experiment in which an external force is applied to the velocity-equilibrated part. Through trial and error it was determined that $T=0.01 \mathrm{~s}, a_{\max }=10000 \mathrm{~cm} / \mathrm{s}^{2}$, and $\mu=0.5$ produced very stable results. Plugging these parameters on Equation 10 yields a predicted value of 1.96 for $K$.

The simulations performed to estimate $K$ consisted in (i) dropping the part on the feeder for 1 second and then applying a force in the $-15,15$ dyn range, and averaging the resulting velocity for the two following seconds. A total of 30 samples in that range were tried (spaced by 1 dyn each), and a linear relationship between $f_{e x t}$ and $\nu^{\prime}$ was measured, as shown in Figure 10. A least-squares fit of the data was performed leading to the equation $y=-4.72+1.95 x$; the measured $K$ is the angular coefficient in this equation, 1.95 which matches within $1 \%$ the analytical value computed above. 

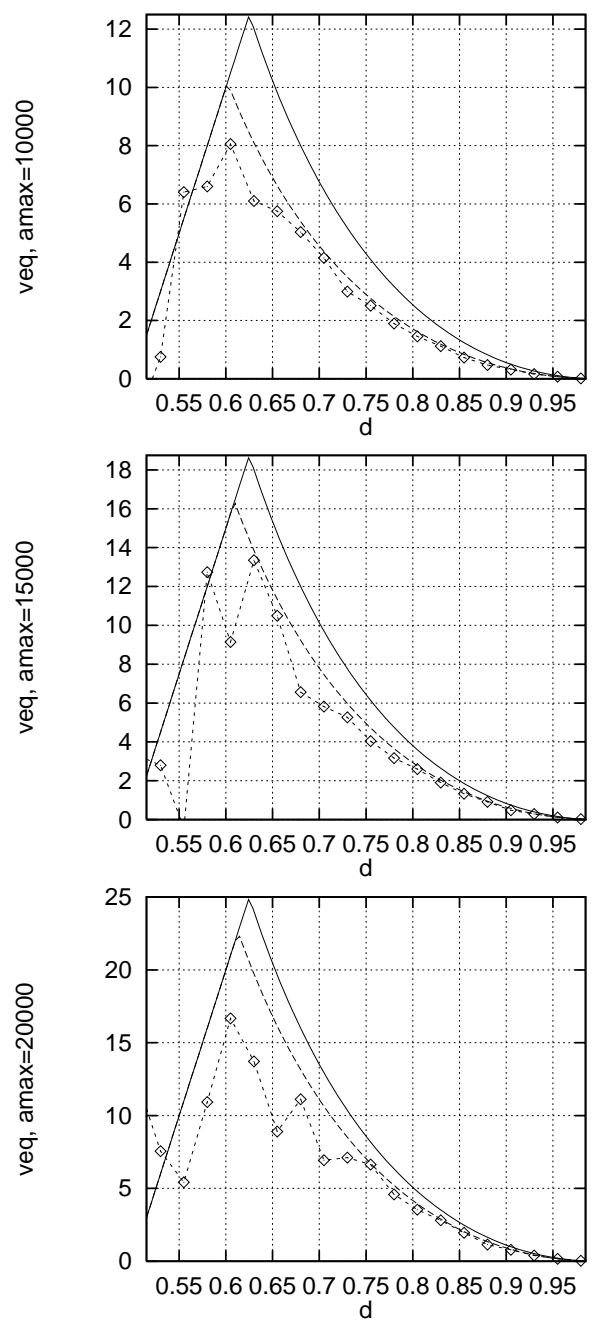

Fig. 9. Simulation results for the part's equilibrium feed rate for $a_{\max }=\{10,15,20\} 10^{3} \mathrm{~cm} / \mathrm{s}^{2}$ (shown from top to bottom).

\section{CONCLUSION}

The dynamics of part motion for a simple longitudinally vibratory parts feeder was studied in this report. Analytical expressions for the feed rate and average frictional forces were derived under both quasi- and non-quasi-static models. These results were verified through rigid-body dynamic simulation. We are currently experimenting with a hardware implementation of the device. Future work will involve comparing the results with experimental measurements, analyzing/measuring $2 \mathrm{~d}$ frictional effects and extending the analysis to parts with an area contact with the feeder. Underway is also a generalization to the current vibration scheme which will allow parallel manipulation of one or more parts over a single vibrating surface.

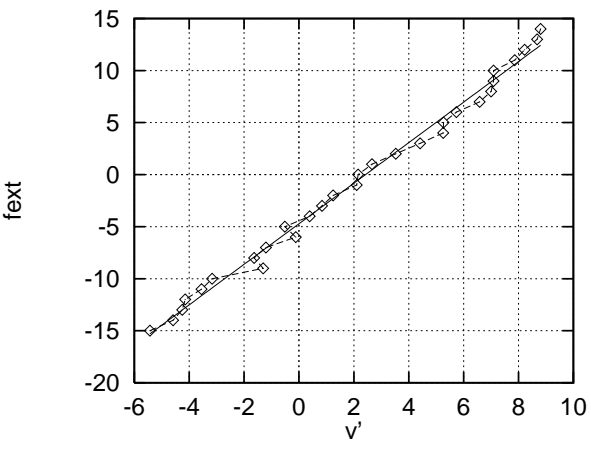

Fig. 10. Plot of $f_{e x t}$ vs. $\nu^{\prime}$, for values of $f$ ext ranging from -15 to 15 dyn, and spaced by 1 dyn. the graph is shown superimposed with the linear function $-4.72+1.95 x$ obtained by a least squares fit of the data points.

\section{REFERENCES}

[1] G. Boothroyd. Assembly automation and product design. Marcel Dekker, Inc., New York, NY, 1991.

[2] Michael Caine. The design of shape interactions using motion constraints. In IEEE International Conference on Robotics and Automation, San Diego, CA, May 1994.

[3] J. Krim. Friction at the atomic scale. Scientific American, pages 74-80, October 1996.

[4] M. Peshkin and A. Sanderson. The motion of a pushed, sliding workpiece. IEEE Journal of Robotics and Automation, 4(6), December 1988.

[5] K. Böhringer, V. Bhatt, and K. Goldberg. Sensorless manipulation using transverse vibrations of a plate. In IEEE International Conference on Robotics and Automation, Nagoya, Japan, May 1995.

[6] S. Konishi and H. Fujita. A conveyance system using air flow based on the concept of distributed micro motion systems. Journal of Microelectromechanical Systems, 3(2):54-58, June 1994.

[7] F. Moesner and T. Higushi. Devices for particle handling by an ac electric field. In IEEE Micro-electromechanical Systems, pages 66-71, January 1995.

[8] J. Luntz, W. Messner, and H. Choset. Parcel manipulation and dynamics with a distributed actuator array: the virtual vehicle. In IEEE International Conference on Robotics and Automation, Albuquerque, NM, April 1997.

[9] J. Suh, S. Glander, R. Darling, C. Storment, and G. Kovacs. Combined organic thermal and electrostatic omnidirectional ciliary microactuator array for object positioning and inspection. In Proc. Solid State Sensor and Actuator Workshop, Hilton Head, NC, June 1996.

[10] K. Böhringer, B. Donald, R. Mihailovich, and N. MacDonald. Sensorless manipulation using massively parallel microfabricated actuator arrays. In IEEE International Conference on Robotics and Automation, San Diego, CA, May 1994.

[11] D. Reznik, S. Brown, and J. Canny. Dynamic simulation as a design tool for a microactuator array. In IEEE International Conference on Robotics and Automation, Albuquerque, NM, April 1997.

[12] B. Mirtich and J. Canny. Impulse-based simulation of rigid bodies. In Symposium on Interactive 3D Graphics, New York, NY, 1995. ACM Press. 